destinée par là-même; à voir se fondèr et se développer chez elle cette industrie nouvelle de l'acide nitrique artificiel.

Précisément, parmi tous les savants et techniciens qui travaillaient un peu partout à la solution de ce problème, se rencontrèrent deux Norvégięns, MM. Birkeland et Eyde, qui surent les premiers, déduire de leurs expériences de laboratoire la possibilité d'une application industrielle, en démontrant que les arcs de grande puissance, à forte intensité,pouvaient être employés en vue de la combustion de l'azote de l'air. Ils fondèrent alors (*), avec l'aide du banquier suédois Wallenberg, une "Société Norrégienne pour l'industrie électrochimique " qui commença à s'assurer, suivant le principe que nous avons indiqué, la possession de plusieurs chutes d'eau et fournit les moyens de construire à Notodden une première petite usine qui fut prète en $\mathrm{I} 905$ avec trois fours de 7 oo HP chacun.
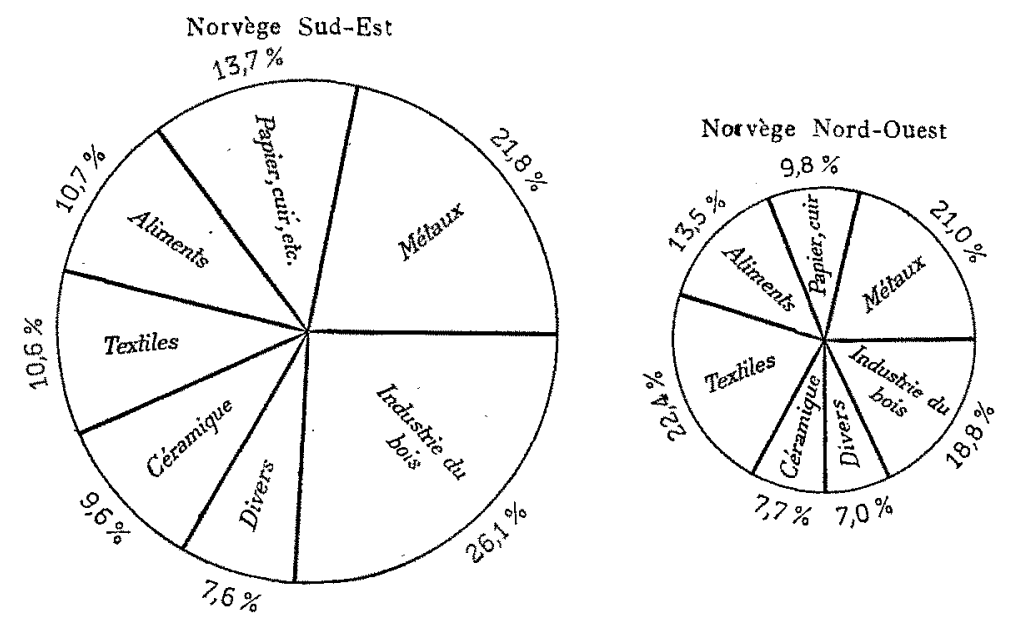

Frs. 2: - Répartition de l'activité industrielle de la Norvège.

Les résultats obtenus donnèrent l'espoir de voir aboutir les recherches dont les dépenses dépassaient déjà I million de couronnes. La fondation d'une nouvelle Société permit d'utiliser la chute de Svolgfos pour installer une fabrique plus importante qui fut prète à la fin de igo7 et qui, après quelques difficultés d'ordre purement électrique au début, fonctionne maintenant normalement.

L'usine de Nottoden comprend 36 fours de $1500 \mathrm{HP}$, dont 32. marchent par la force de Svolgfos, et les 4 autres par celle de Tinfos. Ces fours, du type Birkeland et Eyde, utilisent pour l'allongement de l'arc de forte intensité, le prircipe de Plücker (I86I), d'après lequel l'arc électrique jaillissant entre deux électrodes, placées dans un champ magnétique de direction perpendiculaire à la ligne des électrodes, est étalé en forme de demi-disque qui se forme successivement de chaque côté de la ligne des électrodes, si l'on ernploie le courant, alternatif. L'air arrivant par plusieurs petits canaux, pénètre dans la chambre de réaction qui est un espace rétréci pour assurer une bonne combustion de l'air dont les éléments partiellement combinés sont rapidement entraînés dans un canal périphérique (*). De là, les gaz chauds, après avoir abandonné une partie de leur chaleur dans une chaudière à vapeur, sont encore refroidis jusqu'à $50^{\circ}$ pour passer dans des chambres d'oxydation et destinées à transformer $\Lambda z O$ en $\mathrm{AzO}^{2}$, et traversent enfin plusieurs tours d'absorption, où ils forment successivement de l'acide nitrique au contact de l'eau, et des nitrites et nitrates au contact de la chaux retcnant les dernières traces d'oxyde d'azote.

(*) Conférence de M. Erde à l'occasion des ingénieurs norvígiens (fevrier rooo). Voir La Honille Blavche de septembre roou, page 242 .

(*) Pour plus amnles renseignements sur le procéde Birkeland et Eyde, voir La Houille Blanche d'avril 1908 , page 79 .
On obtient ainsi à Notodden, directément, ou par un traitement ultérieur de l'acide nitrique, une production annuelle de 25000 tonnes de nitrate artificiel, dont le baril de roo kgs est vendu en France i 9 francs, sur wagon RouenMaritime.

Nous avons vu, à propos des installations hydro-électriques, comment s'est établie une entente industrielle entre les procédés et les groupements norvégiens et allemands, avec la création de deux puissantes sociélés travaillant en parallèle, l'une pour l'aménagement des chutes d'eau, l'autre pour la fabrication des nitrates. Le four de la Badische Anilin est d'un principe différent de celui de Birkeland et Eyde : l'arc, de $5 \mathrm{~m}$. de longueur pour un four de 600 IIP, s'établit à l'intérieur d'un tube mélallique relié à l'une des électrodes, et légèrement distant de l'autre électrode, et l'air y circule en tourbillonnant ; le rendement serait, paraîl-il, supérieur à celui du four Birkeland et Fyde et le système de la Badische Anilin serait, par suite, adopté de préférence dans les futures installations de la Norske Salpeterverker qui pourra, dans quelques années, lorsqu'elle sera en possession de 500 ooo HP, lancer sur le marché 300 ooo tonnes d'acide nitrique ou de ses dérivés.

Ce rapide aperçu des ressources, trouvées par la Norvège dans l'utilisation de ses admirables chutes d'eau, suffit pour comprendre le récent développement industriel qui a coïncidé pour ce pays avec l'obtention de son indépendance et d'une situation internationale, dont son commerce extérieur s'est trouvé fortifié.

J. BALIY, Ingénieut des Arts et Mlanufactures.

\section{LA DURANCE ET SON UTILISATION}

(Suite et fin*)

\section{Projet du barrage-réservoir de Serre-Ponçon}

La réalisation du projet du barrage-résecrooir de Gréoulx permettra de remédier à peu près complètement aux pénuries d'eau de la basse Durance. Mais les volumes d'eau qui pourront être emmagasinés dans ce réservoir devront être entièrement réservés au profit des canaux actuellement existants. Le problème de l'extension des irrigations ne sera done pas résolu, et la question des pénuries d'eau industrielles, qui apparait déjà pleine de menaces pour l'avenir, restera entière.

En tenant compte des longs délais nécessaires pour venir à bout des difficultés de toute nature que rencontre un projet de grand barrage-réservoir, il ne semble nullement prématuré de s'occuper dès maintenant des mesures qu'il conviendra de prendre pour satisfaire aux nouveaux besoins, lorsqu'ils se manifesteront d'une façon impérieuse.

Nous avons déjà signalé la rarelé, dans le bassin de la Durance, des emplacements favorables à la création économique de vastes réserves d'eau. En dehors de Serre-Ponçon, et de deux ou trois emplacements dans le bassin du Verdon, nous n'en voyons pas d'autres qui puissent, actuellement, être recommandés pour la construction de grands barrages de retenue d'eau. Les réservoirs qui pourront être établis dans le bassin du Verdon, après celui de Gréoulx, devront être affectés exclusivement au service des irrigations dans ie département du Var. Pour donner satisfaction aux besoins de toute nature qui existent déjà, ou qui se manifesteront

(*) Voir La Houille Blanche de février, mars et mai igro. 
prochainement dans la vallée de la Durance, nous ne voyons qu'une seule solution : la création du barrage-réservoir de Serre-Ponçon.

C'est dans ces conditions que nous avons été amené à reprendre notre ancien projet, et à l'amplifier considérablement, en pous plaçant à un point de vue beaucoup plus général que celui qui était envisagé en 1897 .

\section{PROJET PRIMITIF}

Voici d'abord les dispositions principales du projet que nous avons présenté en rgor. Nous les résumerons très sommairement, car elles sont absolument semblables à celles qui ont été décrites précédemment pour le projet de Gréoulx. Il n'y a guère que les dimensions qui varient d'un projet à l'autre.

Le barrage de Serre-Ponçon qui devait, comme c'est prévu pour celui de Gréoulx, être fondé entièrement sur le rocher, aurait eu une hauteur totale de $95 \mathrm{~m}$., dont $42 \mathrm{~m}$. en fondation, $50 \mathrm{~m}$. de hauteur utile et $3 \mathrm{~m}$. de revanche. La largeur du lit de la Durance à l'emplacement du barrage est de I3o m. environ.

La Durance eât été détournée dans trois souterrains, avec un barrage de garde en amont et une digue de protection en aval. Des murs-écrans, l'un en amont, l'autre en aval de la fouille, devaient être descendus à $10 \mathrm{~m}$. de profondeur dans le gravier.

Malgré l'extrême profondeur du rocher au milieu de la rivière, la fondation à ciel ouvert avec épuisement nous avait paru possible, grâce à une circonstance favorable que nous allons expliquer. Les sondages, au nombre de cinq, que nous avons fait exécuter dans le lit de la Durance, nous ont montré d'une façon absolument concordante que la nature du terrain change à partir d'une profondeur de 7 à $8 \mathrm{~m}$. La couche supérieure est formée par le gros gravier très perméable que l'on voit à la surface du lit. En dessous, et jusqu'au rocher, le terrain est compact, et formé par du gravier à petits éléments avec remplissage des vides par de l'argile. Etant données sa structure et sa composition, ce gravier argileux, qui ressemble à certains dépôts glaciaires, doit êre considéré comme imperméable. Nous étions donc en droit de penser qu'avec les murs-écrans projetés, les infiltrations dans les fouilles seraient peu importantes. Cela ne nous avait d'ailleurs pas empèché, pour plus de sûreté, de prévoir de puissantes pompes centrifuges mues par l'électricité, pour épuiser l'eau dans la fouille.

Le remous du barrage devait s'étendre sur une longueur de $9 \mathrm{~km}$. dans la Durance, et $4 \mathrm{~km}$. dans l'Ubaye. La capacité utile du réservoir eût été de 186 millions de mètres cubes, soit environ le double de celle du réservoir de Gréoulx. Les terrains compris dans le champ d'inondation sont généralement de peu de valeur, et les habitations peu nombreuses. Par contre, nous avions dù prévoir d'importantes rectifications de routes nationales et mème le relèvement, sur une longueur d'un kilomètre, du chemin de fer de Gap à Briançon.

La question des limons et des graviers, qui est encore plus importante que pour le réservoir de Gréoulx, eût été résolue de la même manière.

\section{NOUVEAU PROJET}

Nous devons dire tout d'abord que le nouveau projet n'a été qu'ébauché, notre intention étant de faire ressortir l'intérêt d'une étude approfondie plutôt que d'indiquer une solution ferme et définițive, Néanmoins, au moven des données que nous avons trouvées dans le dossier du projet primitif, il nous a été possible, sur plusieurs points, d'arriver à des conclusions assez précises.

Hauteur de la Retenue. - Une première question, dont l'intérêt est capital, est la fixation de Ja hauteur de la retenue. Nous pensons que celle de $50 \mathrm{~m}$., que nous avions prévue primitivement, doit être notablement augmentée. Il importe, en effet, de donner au réservoir de Serre-Ponçon une capacité utile aussi grande que possible, et cela pour les raisons suivantes :

$I^{\circ}$ Parce que les besoins auxquels il faudra satisfaire deviendront très grands.

$2^{\circ}$ Parce qu'il importe de tirer le meilleur parti possible d'un emplacement merveilleux, qui n'a pas son pareil, à beaucoup près, dans tout le bassin de la Durance.

$3^{\circ}$ Parce que, d'une manière générale, et en particulier dans le cas actuel, la dépense d'établissement d'un réservoir croît moins vite que sa capacité utile. En d'autres termes, le prix par mètre cube de capacité est moins élevé pour un grand réservoir que pour un autre de proportions moindres.

$4^{\circ}$ Parce que les dépenses à faire pour le dévasement du réservoir de Serre-Ponçon seraient sensiblement les mêmes avec une retenue de $50 \mathrm{~m}$. qu'avec une retenue plus grande, et qu'il y a dès lors intérêt à répartir ces dépenses sur une réserve d'eau aussi élevée que possible.

En partant de ce principe, la capacité à donner au réservoir ne devrait, théoriquement, avoir d'autre limite que celle qui correspondrait à une régularisation complète du régine de la Durance. Mais, en pratique, on ne pourrait pas aller aussi loin, et la hauteur de la retenue serait forcément limitée par d'autres considérations. D'une part, en effet, nous estimons qu'en l'état actuel de l'art de l'ingénieur, une retenue de roo $\mathrm{m}$. représenterait un maximum qu'il serait même très audacieux d'atteindre: D'autre part, la considération des terrains à submerger peut aussi conduire à limiter cette hauteur. Il ne saurait, par exemple, être question d'exproprier des communes entières.

C'est cette dernière considération qui intervient pour fixer un maximum à la retenue du barrage de Serre-Ponçon. A I $4 \mathrm{~km}$. en amont de l'emplacement du barrage est situé, au bord de la Durance, le bourg de Savines, chef-lieu de canton issez important du département des Hautes-Alpes. Pour laisser une revanche suffisante entre le plan d'eau maximum du réservoir et les maisons de cette agglomération, il ne faut pas dépasser une hauteur de $85 \mathrm{~m}$. pour la retenue du barrage. Ce chiffre nous paraît d'ailleurs susceptible d'être adopté. Sans doute, il dépasse d'une trentaine de mètres les retenues les plus fortes qui ont été réalisées en France. Mais nous avons l'exemple de l'Amérique, où l'on s'occupe actuellement de construire d'énormes barrages de 80 à $100 \mathrm{~m}$. de hauteur. Il est vrai que, dans notre cas, la difficulté est aggravée par l'extrême profondeur du rocher de fondation. Mais nous indiquerons une solution qui permettra de tourner cette difficulté.

Barrage. - Le barrage aurait une hanteur totale de 4 ? $+85+3=\mathrm{r} 30 \mathrm{~m}$., chiffre qui n'a pas encore été attcint dans le monde entier $\left(^{*}\right)$.

La construction de cet ouvrage gigantesque présenterait donc des difficultés exccptionnelles, mais non insurmontables.

(*) Le barrage actuellement le plus élevé est celui de Shoshone, qui a $100 \mathrm{~m}$. ds hauteur totale, et qui a été décrit dans La Houtlle Blanche de novembre igro. N.D.L.R. 
L'effort maximum de compression dans la maçonnerie atteindra une limite très élevée, de sorte qu'il scra nécessaire, tout au moins vers la base du barrage, de prendre des précautions spéciales.

La partie du barrage qui sera en dessous de l'étiage, c'està-dire celle qui aura à supporter les plus fortes pressions, pourra être constituée par du béton massif et armé, les armatures en fer étant destinées à procurer un grand supplément de résistance, notamment aux efforts de cisaillement. On réalisera ainsi une sorte de rocher artificiel auquel on donnera une résistance aussi grande que l'on voudra.

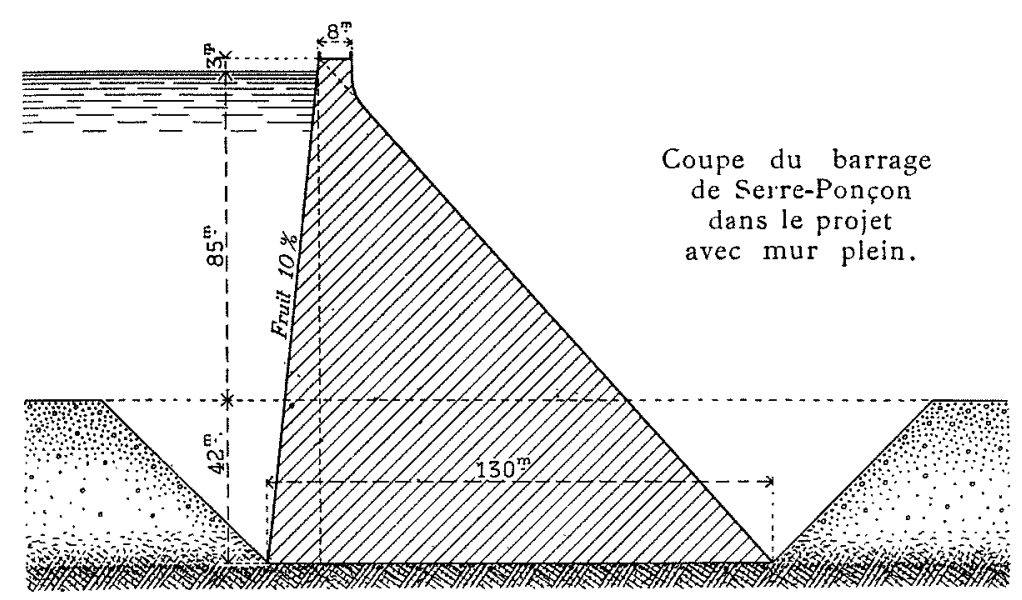

Sur ce massif de fondation, on édifiera ensuite le barrage proprement dit, de $85 \mathrm{~m}$. de hauteur utile. Cet ouvrage pourra être construit en maçonnerie ordinaire, sans que l'on ait à vaincre des difficultés exceptionnelles.

Le mode de construction préconisé pour la base se rapproche quelque peu du béton armé, aussi, de là à adopter carrément le béton armé pour la construction de tout l'ouvrage, il n'y a qu'un pas à faire, et ce pas, la société pour la régularisation de la Durance n'a pas hésité à le franchir. Aussi, tout récemment, vient-elle de se rallier à un projet de barragc en béton armé que nous avions aussi étudié.

En elfet, après mûre réflexion, nous avions acquis la conviction que le béton armé se prête à merveille à la construction de grands barrages de retenue d'eau. En formulant cette opinion, nous nous appuyons d'ailleurs sur la haute autorité de M. Rabut, ingénieur en chef et professeur à l'Ecole des Ponts et Chaussées, qui, au Congrès de 1907 de l'Association Francaise pour l'Avancement des Sciences, a fait une communication sur le béton armé, dans laquelle il a signalé son application à la construction des barrages comme étant appelé au plus grand avenir $\left(^{*}\right)$. D'ailleurs, on a pu voir, dans le précédent numéro de La Houille Blanche, que l'on a déjà construit en Amérique un barrage en béton armé d'une assez grande hauteur.

Le béton armé présente cet avantage important de se prêter tout particulièrement à la réalisation des deux qualités primordiales d'un barrages, qui sont l'étanchéité et la résistance.

La face amont d'un tel barrage est formée par une cloison étanche, plus ou moins inclinée, dont l'épaisseur croît avec la pression de l'eau qui s'exerce sur elle. Des contreforts, convenablement espacés, et reliés par des planchers, supportent cette cloison, et reportent sur le sol de fondation la poussée de l'eau et le poids du barrage lui-même. La pression sur le sol peut être répartie uniformément au moyen d'un radier, également en béton armé comme toutes les autres parties de l'ouvrage. Grâce aux vastes évidements que

( ${ }^{\prime}$ Voir La Houille Blanche de janvier 1908 , p. 16 . comporte le barrage, et à la possibilité de lui donner un grand empattement, la pression par unité de surface sur le sol de fondation pourra être limitée à un chiffre faible, même pour un barrage de très grande hauteur.

L'étanchéité de la paroi amont peut être obtenue d'une manière complète en ajoulant à son armature une âme formée par une tôle, mince et continue, dont les éléments seront reliés par la soudure autogène. Il sera toujours possible de donner à chacun des panneaux de cetle paroi une résistance suffisante contre la poussće de l'eau.

On conçoit, dans ces conditions, qu'un barrage en ciment armé puisse être établi avec toutes les conditions de sécurité désirables. La surveillance d'un tel barrage sera même plus facile et plus efficace que celle d'un barrage en maçonnerie pleine.

En ce qui concerne le barrage qui est projeté à SerrePonçon, l'emploi du héton armé procurera un avantage particulier de la plus grande importance. Grâce à la faiblo pression sur le sol de fondation que donne un barrage de cette nature, pression qui, dans notre cas, ne dépassera guère $6 \mathrm{kgs}$ par $\mathrm{cm}^{2}$, on pourra se dispenser de fonder le barrage sur le rocher. On sait, en effet, que le gravier constitue un sol de fondation résistant et incompressible qui supportera facilement la pression que nous renons d'indiquer.

Il restera à rendre le sous-sol étanche. Une solution absolument sadicale consisterait à descendre en travers de la Durance un mur-écran jusqu'au rocher. Mais nous considérons comme à peu près certain qu'il ne scra pas nécessaire de descendre aussi bas. Si le sous-sol, formé de gravier argileux, est à peu près complètement impermćable comme nous avons lieu de le croire, il suffira de descendre le murécran à une vingtaine do mètres de profondcur.

En tout cas, le radier sur lequel sera construit le barrage ne sera exposé à aucune sous-pression, car la couche de gravier perméable sur laquelle jl reposera constituera un drain puissant.

Cuvette du Réservoir. - Entre Savines et le confluent de l'Ubaye, la Durance coule dans un vaste lit de gravier dont la largeur varie entre 500 et $.000 \mathrm{~m}$., sur un parcours de I3 km. Cette partie de la vallée de la Durance est très peu habitée, et les cultures y sont rares. En la parcourant, on a par moments l'impression de se trouver dans un véritable désert. L'immense plaine de gravier qui forme le lit de la rivière s'étend à perte de vue, dominée par des berges abruptes et dénudées, formées par des rochers et des marnes argilo-calcaires. Au milieu de cette nature sauvage, on rencontre, de distance en distance, quelques habitations entourées de terrains cultivés qui évoquent l'idée de petites oasis : l'Ile de Rousset, la Ribière, le Grand-Pré, le Thubaneau. Seule, la rive gauche de la Durance est boisée sur une partic de sa longueur, mais les bois qui la recouvrent n'ont que peu de valeur. C'est seulement en approchant de Savines que la nature du paysage change,et que les cultures commencent à s'étendre.

Dans la vallée de l'Ubaye la désolation est complète. Sur plus de $4 \mathrm{~km}$., ce ne sont que graviers et rochers nus, sans même un hectare de terrain cultivé.

Rien d'ailleurs ne saurait mieux dépeindre cet état de choses que le tableau des terrains à acquérir qui figure au projet primitif. Le champ d'inondation devait avoir une superficie de 688 hectares, dont $7^{\circ}$ hectares seulement de terrains bien cultivés : vignes, prairies, vergers et labours. Il y en a à peu près autant en terrains de production médiocre, plus 68 hectares de bois taillis et de futaie. Le reste, c'est-à-dire plus de 
5oo hectares, est formé par du rocher nu et des graviers stériles. Dans ce vaste périmètre, on ne trouve que 27 maisons qui ont été estimées ensemble 3 I 5 .0oo francs !

En portant la hauteur de la retenue de 50 à $85 \mathrm{~m}$., on atteindrait, il est vrai, du còté de Savines, des terrains d'une valeur moyenne plus grande, mais pour l'ensemble du périmètre embrassé, dont la superficie est d'environ I 500 hectares, on n'en aurait pas moins des conditions exceptionnellement favorables, qui font de cette partie de la vallée de la Durance un emplacement absolument unique pour l'établissement d'un grand réservoir.

Par contre, les voies de communication seraient fortement touchées. Les routes nationales $n^{\text {os }} 94$ et roo, qui longent la Durance et l'Ubaye, seraient à reconstruire sur plus de $25 \mathrm{~km}$. de longueur. Le chemin de fer de Gap à Briançon devrait être modifié, et rclevé sur une longueur de près de $6 \mathrm{~km}$, à partir d'un point situé à un kilomètre avant la gare de Prunjères qui se trouverait donc complètement submergée.

Il y a licu aussi de se próoccuper du chemin de fer projeté de Chorges à Barcelonnelte, car son tracé passe au milieu do l'emplacement du réservoir, et serait noyé sur une longueur de $9 \mathrm{~km}$. Si donc on devait réaliser un jour le barrage-réservoir de Serre-Ponçon, il importerait de n'avoir pas à rectifier une voie ferrée extrêmement coûteuse. Pour cola, il faudrait établir cette ligne avec un nouveau tracé à déterminer, et pour lequel plusieurs solutions peuvent être envisagées. Si le chemin de fer était établi suivant le premier tracé, toute la partie comprise entre l'origine et la gare d'Ubaye, soit $16 \mathrm{~km}$., devrait être abandonnée, ce qui représenterait une perte d'au moins 9 millions.

Nous dirons enfin que l'examen des lieux, complété par celui de la carle géologique détaillée, montre que la cuvette du réservoir de Scrre-Ponçon doit être complètement élanche.

Capacilé du Réservoir. - Le lac artificiel projeté aurait une longueur de $15 \mathrm{~km}$, avec une largeur atteignant jusqu'à r.500 $\mathrm{m}$. au conlluent de l'Ubaye. Il y aurait en outre unt relenue de $6 \mathrm{~km}$. de longueur dans celte dernière rivière.

Le volume d'eau retenu alteindrait le chifre formidable de 600 millions de mètres cubes. Si énorme que soit ce chilfre, il a cependant été déjà dépassé. Le barrage d'Assouan, sur le Nil, qui a ćté construit il y a quelques années par le gonvernement anglais, retient plus d'un milliard de mètres cubes. En Amérique, on projette actuellement, en vue de l'irrigation d'immenses terrains dans les régions arides de l'Arizona, du Nouveau-Mexique, du Colorado, etc., des réservoirs encore plus importants que celui qu'on pourrait créer à Serre-Ponçon.

D'ailleurs, ce volume d'eau de 600 millions de mètres cubes que l'on pourrait emmagasiner dans le réservoir de Serre-Poncon n'aurait rien d'excessif, eu égard aux besoins à satisfaire ot à l'imporlance du prélèvoment que l'on peut faire sur le débit des hautes eaux de la Durance. Bien que l'on puisse trouver l'utilisation de celte énorme réserve d'eau, nous pensons qu'il serait nécessaire d'en sacrifier une partie dans le but de créer une puissante usine qui serait actionnée par la chute du barrage. 11 importe, en effet, qu'une telle usine ne soit jamais tréduite au chômage, et que la force disponible ne tombe pas en dessous d'un minimum acceptable. Pour satisfaire à cette condition, nous prévoyons que l'on renoncera à utiliser les $40 \mathrm{~m}$. inférieurs de la retenue. On perdra ainsi r ro millions de mètres cubes de capacité utilisable, mais on aura une chute qui atteindra $85 \mathrm{~m}$., et ne tombera jamais en dessous de 40 mètres.

De plus, dans cette partie inférieure du réservoir on pourra pendant fort longtemps loger le produit des dragages qu'il faudra faire. La capacité utile du réservoir sera ainsi ramenée au chiffre encore très respectable de 490 millions de mètres cubes.

Dévasement du Réservoir. - Par comparaison avec les résultats obtenus pour le Verdon, nous avons été amené à évaluer à $200000 \mathrm{~m}^{3}$ au plus le cube annuel moyen des graviers charriés par la Durance. Pour les limons, on pourrait, en partant des chiffres établis pour la Durance à Mirabeau, compter sur un cube annuel de 2 millions de mètres cubes. Les graviers n'ont rien de bien inquiétant, leur cube étant relativement faible. On pourra s'en débarrasser en les draguant, et en les transportant par bateaux dans la partie aval du réservoir. On pourra aussi créer dans des vallées resserrées des barrages de retenue de graviers, par exemple dans les gorges de l'Ubaye, au Lauzet. Quant aux limons, il faudra les refouler de proche en proche dans la partie du réservoir qui sera inutilisée, au moyen de dragues suceuses mues par l'électricité, comme nous l'avons déjà indiqué pour le réservoir de Gréoulx.

Fonctionnement de la retenue. - Les explications que nous avons données sur le régime de la Durance montrent que le remplissage du réservoir de Serre-Ponçon devra s'effectucr principalement au moyen des crues de fonte des neiges, en avril, mai et juin. Les pluies d'automne pourront aussi y contribuer dans une certaine mesure.

Lors de nos études du projet primilif de Serre-Ponçon, nous avions été conduit à rechercher ou à établir les courbes de débits de la Durance, à Rousset, pour une période aussi longue que possible. Nous avons trouvé dans les archives de notre service celles qui avaient été dressées jadis pour la période I $862-1866$. Nous avons établi nous-mêmes celles relatives à la période $\mathbf{I} 892$-I 899 .

L'étude de ces courbes nous a permis d'arriver aux conclusions suivantes : Pendant la période considérée, le débit moyen de la Durance pendant une année a oscillé entre un minimum de $52 \mathrm{~m}^{3}$ en 1893 , et un maximum de $\mathrm{I} 5 \mathrm{r} \mathrm{m}^{3}$ en 1885. Ces débits correspondant respectivement à 1.640 et 4.762 millions de mètres cubes d'eau par an. Pour cette période de treize années, le débit moyen a été trouvé égal à 92 mètres cubes.

Le réservoir de Serre-Poncon, qui serait alimenté précisément par les débits qui sont constatés au pont de Rousset, pcrmettrait de régulariser complètement le débit de la Durance pendant une année sèche, en le maintenant à un chiffre à peu près constant, voisin de $50 \mathrm{~m}^{3}$. Dans une année pluvieuse, le débit resterait variable, mais il serait très amélioré, et les crues seraient fortement atténuées.

Voici maintenant comment nous comprenons le fonctionnement du réservoir. Pendant l'hiver, on utiliserait le volume d'eau emmagasiné pour augmenter le plus possible le débit d'ćtiage, et l'on pourrait conduire la vidange du réservoir de manière qu'elle fủt à peu près terminée vers le 15 avril. I a fonte des neiges commence à se faire sentir à cette époque, parfois même plus tôt, surtout lorsque le temps est pluvieux. A partir de ce moment, on règlera l'écoulement du réservoir à un débit uniforme, variant de 50 à $60 \mathrm{~m}^{3}$ solon l'importance plus ou moins grande du stock de neige dans les montagnes, qui scra déduit approximativement des observations météorologiques.

Habituellement, le remplissage du réservoir sera terminé 
au plus tard dan's les premiers jours de juillet. A partir de ce moment, le debit de la Durance va en diminuant jusqu'aux pluies d'automne, avec cependant quelques petites crues sil se produit de forts orages. On pourra, pendant celte période, maintenir le débit en aval entre 50 et $60 \mathrm{~m}^{3}$, comme ci-dessus. Si les besoins des arrosages l'exigent, on pourra y affecler roo à 120 millions de mètres cubes, et même davantage.

En général, les pluies d'automne permettront de récupcrer ce prélèvement, et c'est avec la réserve totale de 4 go millions de mètres cubes que l'on verra venir l'étiage d'hiver qui s'étend du commencement de décembre à la fin de mars. Dans une bonne année moyeme, on peut admettre que le débit est de $60 \mathrm{~m}^{3}$ au 15 novembre, qu'il décroît en hiver juscru'à $25 \mathrm{~m}^{3}$, et remonte en mars pour dépasser $60 \mathrm{~m}^{3}$ à partir du i5 avril. Pendant cette póriode de cinq mois, le débit moyen sera d'environ 30 à $35^{\circ} \mathrm{m}^{3}$. Avec les 490 millions de mètres cubes disponibles, on pourra l'augmenter d'une moyenne de $35 \mathrm{~m}^{3}$, ce qui portera le débit de la Durance à un taux uniforme de 65 à $7^{\circ}$ mètres cubes.

Prenons maintenant une année très exceptionnelle, telle que rgo8, pendant laquelle il n'y a pas eu de pluies d'automne. Le débit de la Durance est allé en décroissant à pou près régulièrement, depuis le mois de juillet jusqu'en février Igog, pour ne recommencer à croître sérieusement qu'à la fin de mars. Pendant la plus grande partie de l'hiver, le débit n'a guère dépassé une vingtaine de mètres cubes, avec un minimum de $19 \mathrm{~m}^{3}$. Dans cetle période de huit mois, qui va du ${ }^{\text {er }}$ aoùt Igos au 3 I mars Igog, le débit moyen de la Durance a élé d'environ $27 \mathrm{~m}^{3}$. Une réserve de 490 millions de mètres cubes, qui eût été disponible au I $^{\text {er }}$ aont, aurait permis d'augmenter le débit moyen de $22 \mathrm{~m}^{3}$, el de le porter à 49 mètres cubes.

En résumé, on peut dire que le réservoir de Serre-Ponçon permettrait de porter le débit d'étiage de la Durance, à Rousset, à un chiffre de 50 à $70 \mathrm{~m}^{3}$, suivant les années.

Usine de Serre-Ponçon. - Cette usine serait installée immédiatement en aval du barrage, et l'eau du réservoir y serait amenée par un tunnel de dérivation établi en dessous du plan d'eau minimum, qui serait lui-même à $40 \mathrm{~m}$. audessus du niveau actuel de la Durance.

La chute serait comprise entre 40 et $85 \mathrm{~m}$., ainsi que nous l'avons expliqué. Pour tirer le meilleur parti possible de cette chute, il y auraił lieu d'aménager l'usine pour l'utilisation d'un débit de $10 \circ \mathrm{m}^{3}$. La puissance minima de celleci serait de 20 ooo chevaux, avec le débit minimum de. $50 \mathrm{~m}^{3}$ tombant de 40 mètres. La puissance maxima pourrait être portée à 85.000 cheyaux, et la puissance moyenne ne serait pas inférieure à 45.000 chevaux. Cette usine serait donc la plus puissante de tout le bassin de la Durance.

Effet ulile du réservoir de Serre-Ponçon. - Les explications qui précèdent permettent de sàisir, sans qu'il soit besoin d'insister longuement, l'immense utilité que présenterait la création du réservoir de Serre-Ponçon.

En nous plaçant au point de vue des crues, qui a été exclusivement celui des ingénieurs qui ont les premiers étudié la question des barràges de la Durance, vers 1860 , le réservoir de Serre-Ponçon procurerait à lui seul un effet utile plus grand que les six réservoirs qui furent projetés il y a un demi-siècle. Une bónne crue moyenne atteignant un débit maximum de 700 à $800 \mathrm{~m}^{3}$ et représentant un débit moyen de $400 \mathrm{~m}^{3}$ pendant cinq jours, aúgmenterait la réserve de $x 50$ millions de mètres cubes, déduction faite du débit de $60 \mathrm{~m}^{3}$ qu'on laisserait couler en aval. Une telle crue serait facilcment absorbée, soit au printemps soit à l'automne. Une crue plus forte pourrait, suivant les cas, être absorbce, ou tout au moins très atténuée.

Prenons le cas d'une crue exceptionnelle, pouvant àtteindre un maximum de 1.500 à $1.800 \mathrm{~m}^{3}$ par seconde. Daprès l'allure bien connue des graphiques représentant les crues de la Durance, on peut admettre qu'une telle crue aurait un débit moyen de $300 \mathrm{~m}^{3}$ le premier jour, de $x .000 \mathrm{~m}^{3}$ le second, 700 le troisiène, 5 oo le quatrième, etc. Le débit total des quatre premières journées serait de 216 millions de mètres cubes. Aux époques où une semblable crue serait possible, c'est-à-dire du mois d'avril au mois de juin, ou en octobrenovernbre, le réservoir pourrait recevoir de r5o à $400 \mathrm{mil}-$ lions de mètres cubes. Si la crue n'était pas entièrement absorbée, on obtiendrait tout au moins ce résultat capital d'annihiler complètement le flot dangereux, et l'effet de celle suppression pourrait se faire sentir jusque dans la basse Provence. Or, c'est là surtout que les crues sont redoutables. II ne faut pas oublier que lors des crues d'octobre et de novembre 1886 , la belle et riche plaine qui s'étend de Mirabeau au Rhône est restée submergée pendant près d'un mois, et que les dommages causés aux voies de communicalion et aux digues ont dépassé r.200.00o francs. Depuis celle époque, les ouvrages de défense des rives ont, il est vrai, été sérieusement renforcés, mais il n'y en a pas moins un intérêt majeur à diminuer la violence des crues de la Durance.

Fn ce qui concerne les irrigations, les besoins actucls seront à peu près satisfaits par la création du barrage-réservoir de Gréoulx. Mais celui de Serré-Ponçon, en écartant définitivement toute crainte de pénurie d'eau dans la basse Durance, supprimera le principal obstacle qui s'oppose à la dérivation de Fontaine-l'Evêque. De plus, on pourra peu à peu étendre les irrigations comme nous l'avons indiqué.

Mais ce sont surtout les usines qui seront appelées à bénéficier de la régularisation du régime de la Durance qui sera obtenue comme nous l'avons expliqué. En aval du barrage de Serre-Ponçon, et jusqu'au confluent du Buëch, le débit d'étiage, qui est normalement de 25 à $28 \mathrm{~m}^{3}$, et exceptionnellement de $\mathrm{r} 9$ à $2 \mathrm{r} \mathrm{m}^{3}$, sera maintenu habituellement à $60 \mathrm{~m}^{3}$, pour ne descendre probablement jamais en dessous de $50 \mathrm{~m}^{3}$. On pourra donc, dans celte partie de la Durance, établir des usines qui utiliseront, dans de bonnes conditions, un débit de 70 à $80 \mathrm{~m}^{3}$, alors que, avec le régime naturel de la Durance, on ne peut songer à établir les dérivations pour plus de $50 \mathrm{~m}^{3}$, et encore faut-il se résigner à ne marcher pendant quatre à cinq mois par an qu'avec un débit réduit, pouvant descendre pendant trois mois à la moitié de la pleine charge.

Le supplément de débit que déversera dans la Durance le réservoir de Serre-Ponçon profitera d'ailleurs à toutes les usines qu'on pourra établir en aval jusqu'au confluent du Rhône. Comme la Durance à Serre-Ponçon est à l'altitude de $667 \mathrm{~m}$., et qu'à son extrémité aval elle est à une vingtaine de mètres seulement au-dessus du niveau de la mer, il existe entre ces deux points une chute totale de plus de $600 \mathrm{~m}$., qu'il serait possible, au moins théoriquement, de répartir entre diverses usines qui auraient ensemble plus de $500 \mathrm{~m}$. de chute nette. Pratiquement, il est permis d'espérer qu'on arrivera un jour à $400 \mathrm{~m}$. de chute netle totale.

Dans la traversée du département des Hautes-Alpes, entre Serre-Ponçon et la limite des Basses-Alpes, la Durance descend exactement de $19^{2} \mathrm{~m}$. Or, en se reportant au tableau que nous avons dressé des usines construites ou projetées (Valserres, La Saulce, Ventavon et Le Poët), on voit que 
la chute nette totale à réaliser atteint $155 \mathrm{~m}$., ce qui représentera une utilisation de 80 pour ioo.

Il ne paraît pas impossible d'atleindre un résultat approchant pour les parties de la Durance en aval des HautesAlpes.

D’une manière générale, il faut considérer que l'écoulement de la réserve de $49^{\circ}$ millions de mètres cubes devant se faire exclusivement en temps de basses-eaux, soit d'été, soit d'hiver, le supplément de débit qui en résultera pourra êlre utilisé en totalité par les usines. En réalité, si l'on tient compte des prélèvements que l'on pourra faire sur les crues d'automne, c'est sur une réserve annuelle totale d'environ 600 millions de mètres cubes qu'il faudrait tabler en moyenne. L'écoulement de celte énorme masse d'eau représentera, par mètre de chute, une quantité $Q$ d'énergie électrique, exprimée en kilowatts-heures par la formule:

$$
Q=\frac{000.000 .000 \times 1.000 \times 0,7^{5} \times 0,736}{7^{5} \times 60 \times 60}
$$

soit, en nombre rond, $Q=1.200 .000$ kilowatts-heure.

Lorsque les chutes aménagées représenteront un total de 400 mètres, l'industrie bénéficiera d'un supplément d'énergie électrique égal à 480 millions de kilowatts-heures.

Mais ce bénéfice n'est pas le seul que l'industrie aura à relirer de l'opération. Outre l'atténuation des crues, et la diminution du charriage qui en sera la conséquence et facilitera l'entretien des prises d'eau, il y aura aussi une sérieuse amélioration du fonctionnement des dérivations pendant l'hiver. On sait que par les grands froids, la Durance charrie des glaçons qui sont fort gênants, et dont on ne peut se débarrasser à chaque prise d'eau qu'en sacrifiant une partie non négligeable du débit, précisément à l'époque des plus basses eaux. De plus, il y a une tendance à la formation, au sein de la masse d'eau, de particules de glace plus ou moins consistante qui peuvent obstruer les grilles et gêner la marche des turbines.

Le réservoir de Serre-Ponçon permettra de remédier dans une large mesure à cet inconvénient. Au lieu d'avoir en aval du barrage, aux basses températures, de l'eau à $0^{\circ}$ charriant des glaçons, on aura de l'eau à une température voisine de $4^{\circ}$, qui pourra parcourir un long chemin avant qu'il s'y forme des glacons, d'autant mieux qu'en approchant de la Provence le froid sera moins rigoureux. Lorsque les usines prévues dans les Hautes-Alpes fonctionneront, chacune d'elles recevra son cau de l'usine située immédiatement en amont. Ainsi l'eau qui sortira de l'usine de Serre-Ponçon passera directement dans la dérivation de l'usine de Valscrres, et ainsi de suite jusqu'à l'usine du Poët ; chacune de ces usines sera ainsi débarrassée des difficultés et des ennuis que comporte une prise directe dans la Durance, et recevra de l'eau parfaitement décantée, exempte de glaçons, et à une température sensiblement supérieure à $0^{\circ}$, de telle sorte que, avec le tirant d'eau de 3 à $4 \mathrm{~m}$. des dérivations, on n'ait absolument rien à craindre des froids les plus intenses.

Dépenses. - Nous n'avons pas la prétention de donner dès maintenant une évaluation précise de la dépense. Cependant, en nous basant sur les estimations rectifiées du projet primilif, et en établissant largement nos prévisions, nous croyons pouvoir dire que la dépense totale du barrage-réservoir de Serre-Ponçon ne dépassera pas 5o millions, y compris les acquisitions de terrains et le rétablissement des voies de communication.

Cette dépense serait largement justifiée par les avantages de toute nature que procurerait le réservoir de Serre-Ponçon.
La location de la chute du barrage, à raison de 20 francs par poncelet de force brute moyenne, chiffre qui n'a rien d'exagéré, produirait, pour les 45.000 chevaux susceptibles d'être développés, goo.0oo francs par an.

La puissance supplémentaire qui sera fournie aux usines d'aval vaudra pour le moins un centime par kilowatt-heure, ce qui, pour les 480 millions de kilowatts-heures prévus, représenterait annuellement 4.800 .000 francs.

La seule considération des forces hydrauliques fait donc ressortir un bénéfice qui pourra atteindre 5.700.000 francs par an.

Les avantages d'ordre agricole, qu'il serait plus difficile d'évaluer en argent, seront certainement très importants.

Les chiffres que nous venons d'indiquer établissent d'une façon indiscutable l'utilité de la création du réservoir de Serre-Ponçon.

\section{Résumé et Conclusions}

Nous avons essayé de donner un aperçu de l'utilisation actuelle des eaux de la Durance. Nous avons montré les résultats magnifiques qui ont été obtenus par l'irrigation, et nous avons mentionné les débuts de l'industrie nouvelle de la houille blanche. Que de changements cela représente depuis l'époque lointaine où la Durance, d'après un dicton populaire,était considérée comme un des fléaux de la Provence, concurremment avec le mistral et le Parlement ! Les siècles ont passé, et le parlement a disparu. Mais si le mistral nous est resté, il y a déjà longtemps que la Durance n'est plus un fléau. Le génie de l'homme, après avoir endigué le redoutable torrent alpin, a dérivé ses eaux vers de vastes territoires qui étaient stériles et desséchés, et leur a apporté la vie et la prospérité.

L'œuvre qui a été réalisée est considérable, mais elle n’est pas terminée. Les irrigations ont pris un tel développement que l'eau de la Durance devient souvent insuffisante, et cependant il y a de nouveaux besoins qui se manifestent. L'industric de la houille blanche vient à peine de naître, et déjò elle réclame une régularisation du régime de la capricieuse rivière. La même question se pose d'ailleurs plus ou moins pour toutes les rivières des Alpes, car partout l'industrie des grandes forces hydrauliques souffre du manque d'eau, surtout pendant l'hiver. C'est donc à juste titre que l'on se préoccupe de remédier à cette fâcheuse situation. Malheureusement, il sera le plus souvent impossible d'arriver à une solution pratique de ce problème si complexe, mais c'est une raison de plus pour agir là où les circonstances sont favorables. Nos forces hydrauliques des Alpes représentent un élément important de la richesse publique, et il y a un intérêt des plus sérieux à les mettre en valeur.

Jusqu'à présent, les tentatives de régularisation de ces cours d'eau ont été assez rares.

Aristide Bergès, qui a été le promoteur de l'industrie de la houille blanche dans le Dauphiné, a, le premier, montré le parti que l'on peut tirer d'un petit cours d'eau en uniformisant son débit. Par l'aménagement d'un minuscule lac de montagne, il est parvenu à assurer une force motrice à peu près constante à son usine de Lancey, qui est actionnée par une chute de $500 \mathrm{~m}$. de hauteur.

D'autres améliorations de même nature seront certainement réalisées.

Le projet Dalloyau, dont nous avons dit quelques mots, prévoit aussi l'utilisation de forces motrices par la régularisation du Verdon.

Enfin, le haut Rhône nous fournit un exemple remarquable dans le même ordre d'idées. La nature, il est vrai, a sin-. 
gulièrement facilité le travail de l'homme, car il a suffi de créer à la sortie du lac Léman une retenue de un mètre de hauteur pour obtenir une réserve de 582 millions de mètres cubes, qui a permis de tripler le débit d'étiage naturel du Rhône en aval.

Quoi qu'il en soit, les efforts qui ont été faits depuis une dizaine d'années, en vue d'améliorer et de développer les irrigations en Provence, paraissent sur le point d'aboutir à un double résultat : la création du barrage-réservoir de Gréoulx, qui mettra fin aux pénuries d'eau de la basse Durance, et l'aménagement du lac d'Allos, qui rendra possible, ou tout au moins facilitera dans une importante mesure la dérivation de Fontaine-l'Evêque.

Mais les résultats qui seront ainsi obtenus, quoique très importants, ne seront pas encore suffisants pour arriver à une solution à peu près complète des problèmes qui sont actuellement posés.

La suppression des pénuries d'eau, et l'extension éventuelle des arrosages dans le département des Bouches-duRhône, la dérivation de Fontaine-l'Evêque qui permettra d'amener de l'eau potable à Marseille et à Toulon, et d'entreprendre des irrigations dans le Var, l'aménagement et l'utilisation des forces hydrauliques de la Durance, qui intéressent à un haut degré la prospérité industrielle de Marseille et de la vallée du Rhône, toutes ces entreprises sont connexes, et liées étroitement à la régularisation du régime de la Durance et de son principal affluent le Verdon. Même les irrigations projetées dans plusieurs départements de la rive droite du Rhône y sont intéressées, car elles doivent être assurées au moyen de l'eau de ce fleuve, qui sera élevée mécaniquement par des pompes actionnées par de l'énergie électrique à très bon marché, empruntée aux usines de la Durance.

Pour réaliser complètement ce programme, il faudra non seulement faire les réservoirs d'Allos et de Gréoulx, mais il faudra encore créer un important réservoir sur la Durance elle-même. Or, il existe sur cette rivière, à Serre-Ponçon, un emplacement merveilleux, unique même, pour la création d'une énormé retenue d'eau. Au moyen d'un barrage de $85 \mathrm{~m}$. de hauteur utile, on aurait une réserve de $490 \mathrm{mil}-$ lions de mètres cubes qui permettrait d'augmenter le débit d'étiage de la Durance de 30 à $35 \mathrm{~m}^{3}$ par seconde.

Avec cette puissante réserve d'eau, dont le renouvellement chaque année sera largement assuré,on apportera un remède définitif aux pénuries de la basse Durance. Si le réservoir de Gréoulx devait ne pas y suffire complètement, l'appoint nécessaire serait demandé au réservoir de Serre-Ponçon, qui permettrait en outre l'extension des arrosages. On mettrait fin ainsi au conflit qui divise depuis longtemps les départements du Var, des Bouches-du-Rhône et de Vaucluse. Ces deux derniers, qui s'opposent actuellement à la dérivation de Fontaine-l'Evêque, n'auront plus aucune raison pour le faire lorsqu'il existera une énorme réserve d'eáu qui sera de nature à rassurer les esprits les plus inquiets et les plus défiants.

Pour achever de dégager la question de Fontaine-l'Evêque il serait utile, il est vrai, de faire tomber l'opposition de la ville d'Aix, et des usagers du canal du Verdon, qui ne bénéficieraient en rien des avantages que procureraient les réservoirs de Gréoulx et de Serre-Ponçon. Mais si le réservoir du lac d'Allos devait se montrer insuffisant, on pourrait en faire un autre, par exemple celui de Caréjuan, qui est d'ailleurs compris dans le projet Dalloyau.

En ce qui concerne les forces hydrauliques, la création du barrage de Serre-Ponçon présenterait également des avantages très importants. Au barrage même, on pourrait créer une usine dont la puissance atteindrait 85.000 chevaux, avec une moyenne de 45.000 chevaux. De plus, le débit d'étiage de la Durance, jusqu'à Avignon, sur un parcours de $220 \mathrm{~km}$., serait notablement augmenté. On mettrait ainsi à la disposition de l'industrie une quantilé d'énergie supplémentaire, qui serait d'au moins 480 millions de kilowattsheure par an, ce qui faciliterait beaucoup la création des usines le long de la Durance.

Enfin, les dangers d'inondation seraient à peu près complètement supprimés jusqu'à Sisteron, et bien diminués au delà.

En résumé, la création du barrage-réservoir de Serre-Ponçon présenterait d'immenses avantages pour l'agriculture et l'industrie dans une grande partie de la région du SudEst de la France, et il est à désirer que cet important projet puisse un jour être réalisé.

Pour les amateurs de pittoresque, le lac artificiel de la Durance constituera une attraction peu ordinaire, dans un cadre sauvage et grandiose. Nombreux seront les touristes qui viendront parcourir ses rives, ou naviguer sur ses eaux bieues, dans lesquelles se mireront les hautes cimes du Morgon et du Colbas.

La dépense à faire, qui peut être évaluće à 50 millions, ne sera nullement exagérée, eu égard au but à atteindre. Elle serait couverte par les avantages de toute nature que l'on obtiendrait, et notamment par la location de la chute du barrage, et par les redevances que paieraient les arrosants et les industriels intéressés.

Avant que la construction du chemin de fer de Chorges à Barcelonnette ne vienne rendre presque impossible la réalisation du projet, dont nous venons de tracer les grandes lignes, nous estimons que nous avions le devoir de le faire connaître.

Tel est le but principal que nous avions en vue en publiant cette étude.

I. WILHELM.

Ingénieur en Chef des Ponts-et-Chaussées.

\section{THERMODYNAMIQUE}

\section{A PROPOS DU FROID INDUSTRIEL}

Il y a cinq ans (nov. I go5), je rendais compte, ici même, des leçons professées à Bordeaux par M. Marchis sur le froid industriel, ef ce m'était une occasion de rappeler, après lui, l'attention sur M. Ch. Tellier, le véritable initiateur de l'industrie du froid.

Depuis cette époque a eu lieu, à Paris, en octobre r go8, le premier Congrès international du froid industriel, au. cours duquel 6 ooo congressistes venus de tous les points du monde saluèrent dans un enthousiaste ćlan Ch. Tellier du titre de Père du froid: J'y étais, et j'affirme que la scène ne manquait pas de grandcur ! Ce jour-là le vieux savant, lo vieil apôtre, ignoré dans son propre pays qu'il avait voulu enrichir el qui ne le paya que d'ingratitude et d'oubli, le vieux chercheur modeste et probe put se convaincre que, si la foule ne le connaissait pas et le coudoyait sans ménagement, les gens capables de l'apprécier lui vouaient, par contre, sur la terre entière, toute leur gratitude et toute leur admiration.

Il y avait aussi, il faut bien le dire, dans cette ovation, 